\title{
The role of the Advanced Therapies initiative
}

\author{
Enrico Bosone \\ Regulatory Affairs \& Compliance, Celgen, Italy \\ E-mail: ebosone@celgene.com
}

The European Regulation on "Advanced Therapies"- covering gene therapy, somatic cell therapy and tissue engineered products - is the newest European Regulation and deals with three scientific fields which induce high expectations of valuable benefits.

The complexity of these three areas with the current technical and scientific difficulties has slowed down the wished speed of their development. However the few successes obtained so far are so impressing that the intention and the will to pursue this option for novel therapies are increasing.

Some real examples of these results, or of the promises of results, have been reported: the gene therapy for ADA-SCID immunodeficiency, the impressive results of Anginera in the cardiac ischemia and the aims of the project regarding a gene therapy approach for the treatment of $\beta$-thalassemia major, are few but outstanding cases.

In addition to the scientific difficulties in the development of this therapeutic approach, many "Advanced Therapies" projects suffer from weak protection of intellectual properties. Some "Advanced Therapies-like" procedures have already been applied in some Medical Centres in Europe: the Health Authorities' control, together with the recently instituted CAT, (Committee for Advanced Therapies), particularly sensitive and protective towards real innovation, should guarantee the right rewards.

However, the availability of special funding from charities and Patient Associations, together with ad hoc incentives from the Community seems compelling to foster research in the area and make the development of AT feasible. The first steps of CAT seem to move in the right direction. 